\title{
Electrically enhanced drying of white champignons
}

\section{Bashkir, I.; Kudra, T.; Martynenko, A.*}

Department of Engineering, Faculty of Agriculture, Dalhousie University, Truro, NS B2N, Canada

*E-mail of the corresponding author: alex.martynenko@dal.ca

\begin{abstract}
Electrohydrodynamic (EHD) drying is a novel non-thermal dewatering technology using electric discharge in air to enhance dehydration of heatsensitive biomaterials. Low energy consumption and high product quality make it attractive for industry. In this study, the mushrooms slices have been dried under sole EHD with 12, 14, and $16 \mathrm{kV} \mathrm{DC}$, and EHD in combination with air cross-flow at $1 \mathrm{~m} \mathrm{~s}^{-1}$ and different relative humidity $(\mathrm{RH})$ from 30 to $70 \%$. It was found that drying kinetics was exponential over initial moisture contents from 3.3 to $14.1 \mathrm{~g} \cdot \mathrm{g}^{-1}(\mathrm{db})$. The equilibrium moisture content ranged from 0.15 to $0.1 \mathrm{~g} \mathrm{~g}^{-1}$ depending on $R H$. Decrease of $R H$ or air cross-flow significantly improved efficiency of EHD drying.
\end{abstract}

Keywords: mushrooms; electrohydrodynamic; ionic wind; air humidity; equilibrium moisture content. 


\section{Introduction}

Electrohydrodynamic (EHD) drying is regarded as a non-thermal dewatering technology, suitable for drying of heat-sensitive materials because of high product quality and low energy consumption. ${ }^{[1]}$ Hence, it is excellent alternative as compared to hot-air convective drying of mushrooms with extremely short shelf life, which should be consumed or processed within hours after harvest. ${ }^{[2]}$

Commonly accepted hypothesis is that EHD drying occurs due to ionic wind. The effect of ionic wind on shiitake mushrooms drying was first investigated by Xue et al ${ }^{[3]}$ under 6, 8, $10,12 \mathrm{kV}$ AC (electric field strength from 3 to $6 \mathrm{kV} \mathrm{cm}^{-1}$ ), concurrent airflow at $45^{\circ} \mathrm{C}$ and $40 \% \mathrm{RH}$. The results showed that electro-convective drying for 10-14 hours was faster by 1.1 to 1.6 times compared to control. The effect of ionic wind increased with increasing voltage and decreased with increasing airflow rate. However, slight browning of the dried shiitake gills under the effect of ionic wind was noticed. Dutta et al. ${ }^{[4]}$ showed that pretreatment of mushroom slices with AC electric field $4.3 \mathrm{kV} \mathrm{cm}^{-1}$ accelerated subsequent convective or freeze drying. They also reported that the EHD pre-treatment improved quality of dry mushrooms, in particular lesser shrinkage, higher rehydration ratio and enhanced color as compared to control. Dinani et al. ${ }^{[5,6]}$ investigated the effect of EHD in combination with hot air drying at $60^{\circ} \mathrm{C}$ and $10 \% \mathrm{RH}$ for voltages 17,19 and $21 \mathrm{kV}$ DC, and gaps between electrodes 5,6 and $7 \mathrm{~cm}$. Electric field accelerated drying rate by 1.38, 1.41 , and 1.43 times for voltages, and by $1.52,1.36$, and 1.33 times for gaps, so one can conclude that increasing of electric field strength significantly increased water diffusion in mushrooms. These experiments were done under conditions of natural convection. Similar experiments with EHD in combination with hot-air drying at $45^{\circ} \mathrm{C}$ under conditions of forced convection ${ }^{[7-9]}$ showed significant effect of air velocity. The enhancement of the drying rate due to EHD was as high as $1.40,1.56$, and 1.78 times at $0.4 \mathrm{~m} \mathrm{~s}^{-1}$, while at 2.2 $\mathrm{m} \mathrm{s}^{-1}$ these were 1.08, 1.03, and 0.97 times (no significant effect of EHD). These results confirmed previous conclusions of Xue et al. ${ }^{[3]}$ that EHD is efficient only under low air velocities, while increase of air velocity above certain threshold results in purely convective drying. Unfortunately, all these experiments with mushrooms were conducted for combination of EHD with hot air drying with temperature as the dominant drying factor. These settings hindered the role of EHD in drying, making difficult interpretation of sole EHD effect on drying kinetics and product quality.

Promising experiments on EHD mushroom drying confirmed the importance of industrial scaling of the EHD technology. This research was initiated on the request of industry partner Jantex Ltd (Poland) to improve efficiency and quality of white champignons drying because of high sensitivity of product color to drying temperature. The main objective of this paper is to present preliminary results of EHD drying of sliced white champignons, including the effects of relative humidity, air cross-flow, initial moisture content, electric 
field strength and configuration of discharge electrode, which are instrumental for industrial scaling of EHD technology.

\section{Materials and methods}

White champignons (Highline, Leamington, Canada) purchased from a local grocery were cut perpendicularly to the head into 5-mm slices and then spread uniformly in a single layer on the collecting electrode. Initial mass of sliced champignons varied from 30 to $34 \mathrm{~g}$. Initial moisture content was in the range 3.3 to $14.1 \mathrm{~kg} \mathrm{~kg}^{-1}(\mathrm{db})$.

Experimental apparatus is shown in Figure 1. The drying chamber (1) was made of Plexiglas $(40 \times 20 \times 20 \mathrm{~cm})$ with two openings $10 \mathrm{~cm}$ in diameter. Discharge electrode (2) was built from 143 needles arranged into $11 \times 13$ rows giving $1 \times 1 \mathrm{~cm}$ needles packing density or 42 needles arranged into $6 \times 7$ rows giving $2 \times 2 \mathrm{~cm}$ needles packing density. Discharge electrode was connected to the positive pole of a DC high voltage source, while aluminum plate (3) with the size $(20 \times 10 \mathrm{~cm})$ was used as a grounded electrode. Both electrodes were placed in the drying chamber. The room air humidity was maintained at $20 \%$ by the air conditioning system. Airflow velocity was maintained at $1.0 \mathrm{~m} \mathrm{~s}^{-1}$ by a 1.5 W fan (5) and measured with the hot wire anemometer HD300 (Extech Instruments, USA). High voltage (6) was supplied by DC power supply BAL-32-5 (Voltronics, USA). Voltage and current were recorded continuously using the LabView2012 (National Instruments, USA) data acquisition system with USB-6120 interface and desktop computer (7). The weight of mushrooms was determined with a digital scale (4) (model HCB 1002, Adam Equipment, Oxford, CT, USA).

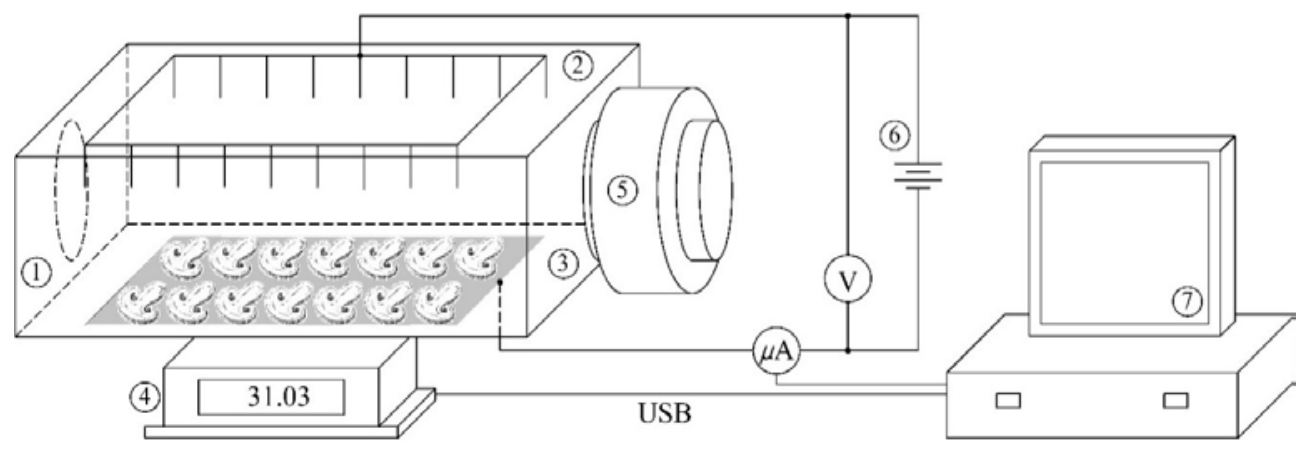

Fig. 1 EHD experimental setup.

Experiments for determination of equilibrium moisture content were carried out for sole EHD at 12, 14, and $16 \mathrm{kV}$ with a gap $3.0 \mathrm{~cm}$ between electrodes, or EHD combined with air cross flow of $1.0 \mathrm{~m} \mathrm{~s}^{-1}$. The effect of initial moisture content on EHD drying rate was measured for fresh slices and for slices after storage in the cooler from one to three days. The effects of EHD (1) and EHD + air cross-flow (2) were compared with sole air cross- 
flow (control 1) or natural convection (control 2) for the same batch of slices. Drying rate for each treatment was calculated for 30 min using four-steps drying protocol. For these experiments the $\mathrm{RH}$ was kept constant at $20 \pm 2 \%$ and room temperature of $20 \pm 0.5^{\circ} \mathrm{C}$.

\section{Results and discussions}

The effect of discharge electrode geometry and air humidity on drying kinetics is shown in Figure 2 . The effect of $1 \times 1$ and $2 \times 2 \mathrm{~cm}$ needle packing density was studied at $15 \mathrm{kV}, 3.5$ cm gap, RH 70-80\% and air cross-flow $0.4 \mathrm{~m} \mathrm{~s}^{-1}$ (Fig. 2A). Drying kinetics showed the difference between effects of two electrodes. In particular, $1 \times 1$ electrode initiated linear drying kinetics (constant drying rate), which indicates convection-limited conditions. In contrast, $2 \times 2$ electrode initiated exponential drying kinetics (falling drying rate), which indicates diffusion-limited drying. Both cases have been reported in literature,$^{[5,10]}$ however the reason for the difference in drying behaviour has not been discussed.

Better results of EHD drying, obtained with $2 \times 2$ electrode, determined preferable use of such electrode in further experiments. The effect of 30,50 , and $70 \%$ relative humidity on the drying kinetics was studied with $2 \times 2$ electrode at $22 \mathrm{kV}, 3.5 \mathrm{~cm}$ gap (electric field 6.3 $\mathrm{kV} \mathrm{cm}^{-1}$ ) and superficial velocity of air cross-flow $1.0 \mathrm{~m} \mathrm{~s}^{-1}$ (Fig. 2B).


Fig. 2 Moisture kinetics for different discharge electrodes (A) and relative humidity (B).

Drying time was in the range from 7 to 20 hours, depending on relative humidity. The equilibrium moisture content at the end of drying was in the range from 0.1 to $0.15 \mathrm{~g} \mathrm{~g}^{-1}$. This moisture content for white champignons corresponds to the range of water activities $\mathrm{a}_{\mathrm{w}}$ from 0.3 to $0.35^{[11]}$, which is considered as safe for shelf-life stability. ${ }^{[12]}$ These data demonstrate how important is to maintain low air relative humidity in the EHD drying.

Effect of sole EHD and EHD combined with convective air cross-flow on drying kinetics is presented in Figure 3. It follows that drying rate increases with voltage, which corresponds to results reported earlier. ${ }^{[8,9]}$ Drying time to reach $0.2 \mathrm{~g} \mathrm{~g}^{-1}$ of moisture content (dashed line) significantly decreased from $25 \mathrm{~h}$ at $4.0 \mathrm{kV} \mathrm{cm}^{-1}$ to $12 \mathrm{~h}$ at $5.3 \mathrm{kV} \mathrm{cm}^{-1}$. Effect of combined air cross-flow and ionic wind was significant at 12 and $14 \mathrm{kV}$, however at $16 \mathrm{kV}$ 
the drying effect of sole ionic wind was higher. This result is difficult to explain within existing theories of EHD drying. Therefore, the phenomenon of interaction between ionic wind and air cross-flow requires additional research.

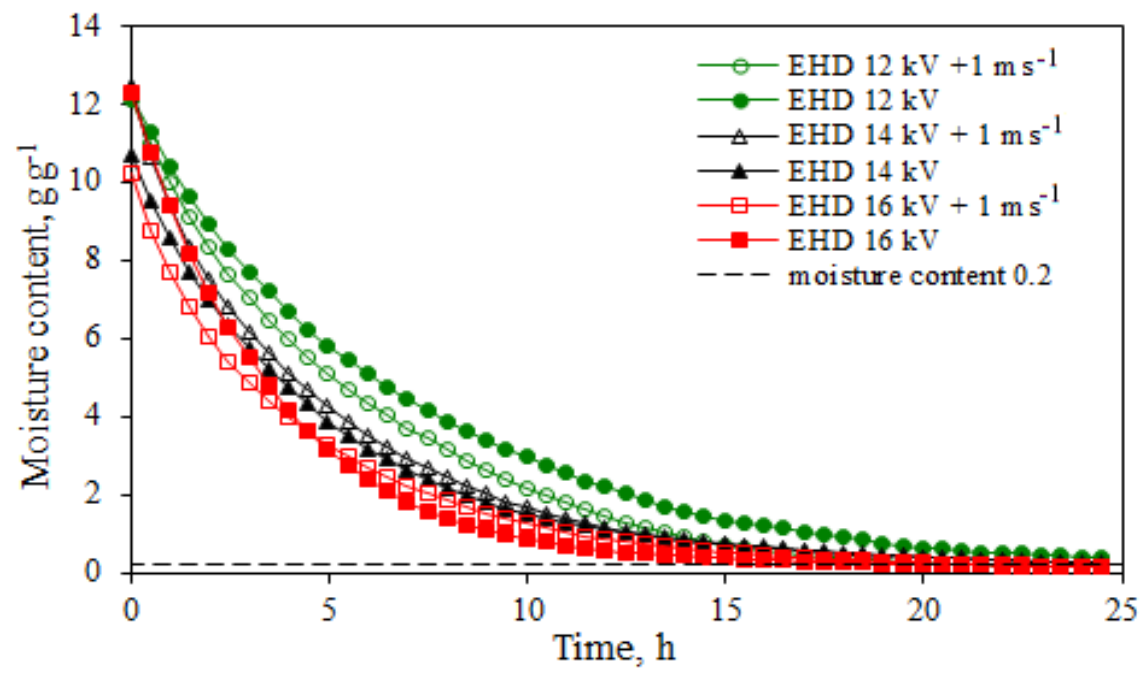

Fig. 3 Effect of EHD drying and combined EHD with convective cross-flow drying (1.0 ms $\left.{ }^{-1}\right)$ under different voltages on drying time (3 cm gap, 20\% RH).

The effective moisture diffusivity $\left(D_{\text {eff }}\right)$ can be calculated from the following equation:

$$
D_{\text {eff }}=\ln \left(\frac{\pi^{2}}{8} M R\right) \cdot\left(\frac{4 L^{2}}{\pi^{2} t}\right)=(-0.0851-0.4053 \ln M R) \cdot \frac{L^{2}}{t}
$$

Based on equation (1) it is possible to estimate instantaneous diffusion coefficient from experimentally determined parameter $k$ :

$$
D_{\text {eff }}=\left(-0.0851 \cdot \frac{1}{t}+0.4053 k\right) \cdot L^{2}
$$

Results of experimental evaluation of $k$ for different modes of EHD drying are presented in Figure 4. Parameter $k$ was stable over drying period from 0 to 14 hours in the wide range of voltages with/without forced convection, which indicates a suitability of the exponential model for approximation of drying kinetics in EHD drying. Based on the average value of parameter $k$, the effective moisture diffusivity coefficient has been calculated (Table 1):

Table 1. Effective moisture diffusivity coefficient, $D_{\text {eff }} \cdot 10^{-10} \mathrm{~m}^{2} \mathrm{~s}^{-1}$

\begin{tabular}{lccc}
\hline \multicolumn{1}{c}{ Drying } & $\mathbf{1 2} \mathbf{~ k V}$ & $\mathbf{1 4} \mathbf{~ k V}$ & $\mathbf{1 6 ~ k V}$ \\
\hline EHD & $3.29 \pm 0.31$ & $4.84 \pm 0.11$ & $6.42 \pm 0.30$ \\
EHD $+1.0 \mathrm{~m} \mathrm{~s}^{-1}$ & $3.78 \pm 0.23$ & $5.09 \pm 0.14$ & $5.39 \pm 0.20$ \\
\hline
\end{tabular}




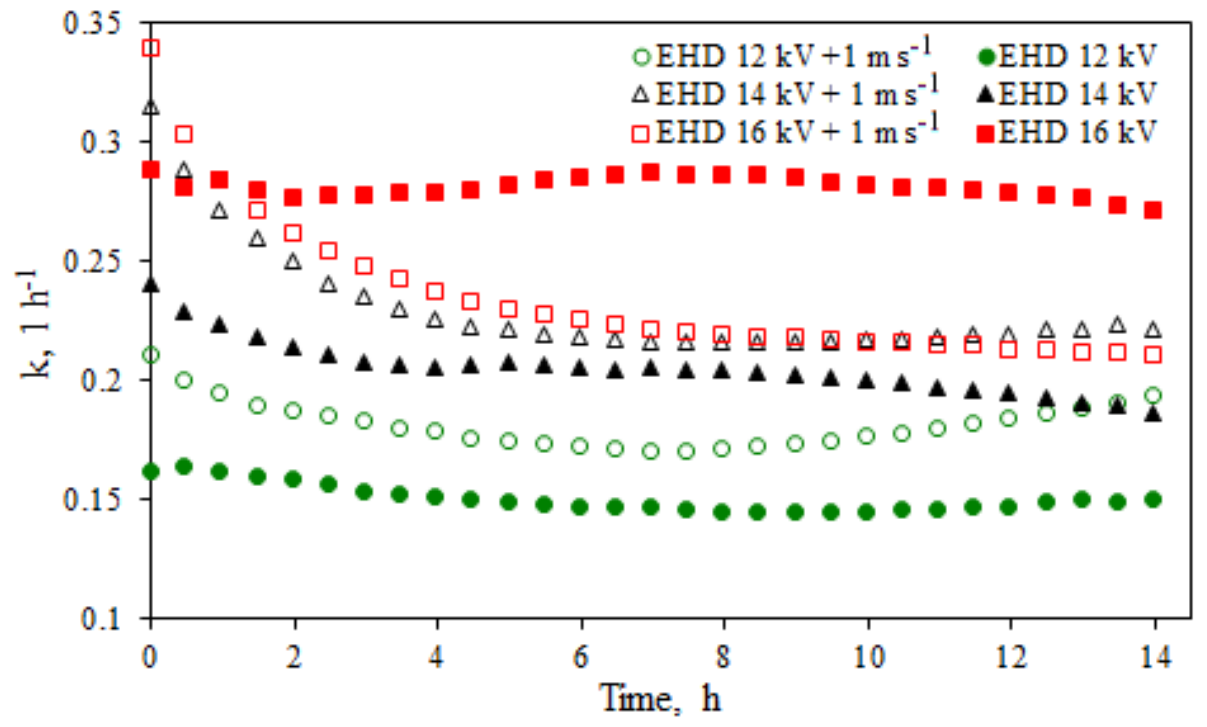

Fig. 4 Velocity of EHD drying and EHD + convective cross-flow drying $\left(1.0 \mathrm{~m} \mathrm{~s}^{-1}\right)$ under different voltages on drying time.

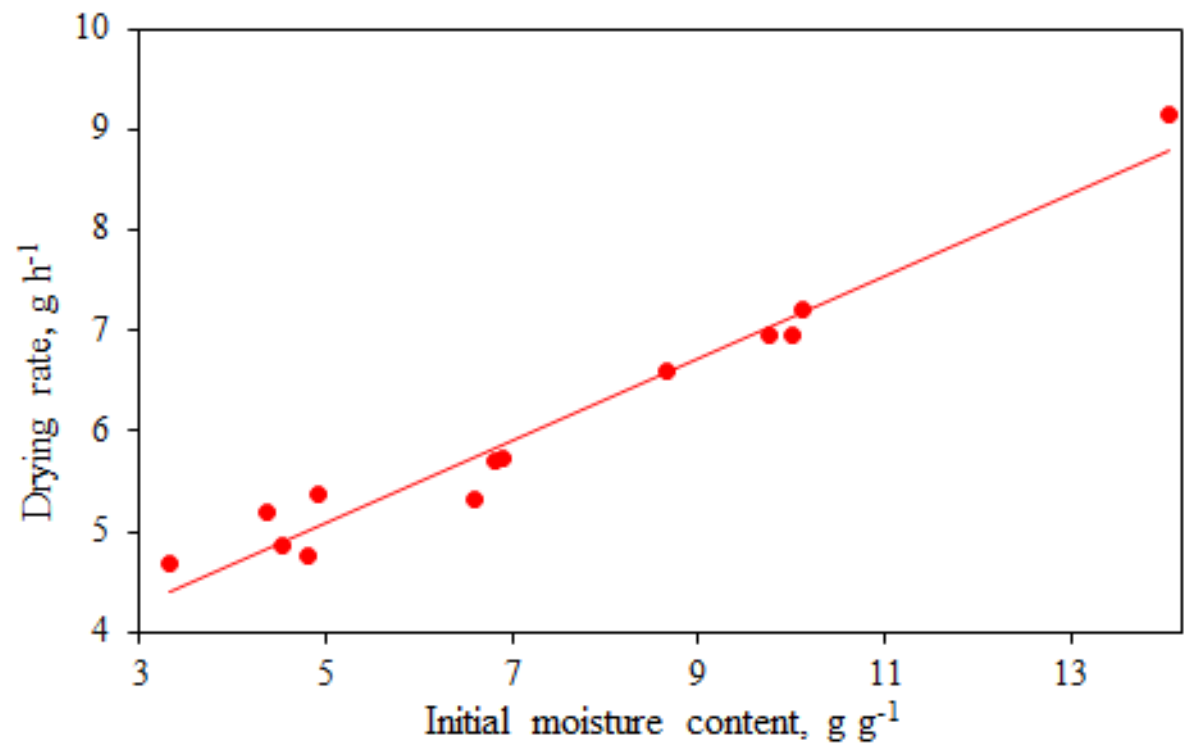

Fig. 5 Dependence of drying rate (DR) on initial moisture content $\left(X_{0}\right)$ at EHD $14 \mathrm{kV}$ and $\mathrm{RH} 20 \%$ (fitted linear regression $D R=3.056+0.4087 X_{o}$ with $R^{2}=0.962$ )

From these results it is clear that drying rate is proportional to moisture content, which validates initial assumption, underlying simplified diffusion model. To study interactions between forced air cross-flow and electro-convection, the four-step experimental protocol 
was designed, exploring three cases in various combinations: (1) Sole EHD, (2) air crossflow, and (3) EHD with simultaneous air cross-flow (Fig. 6).

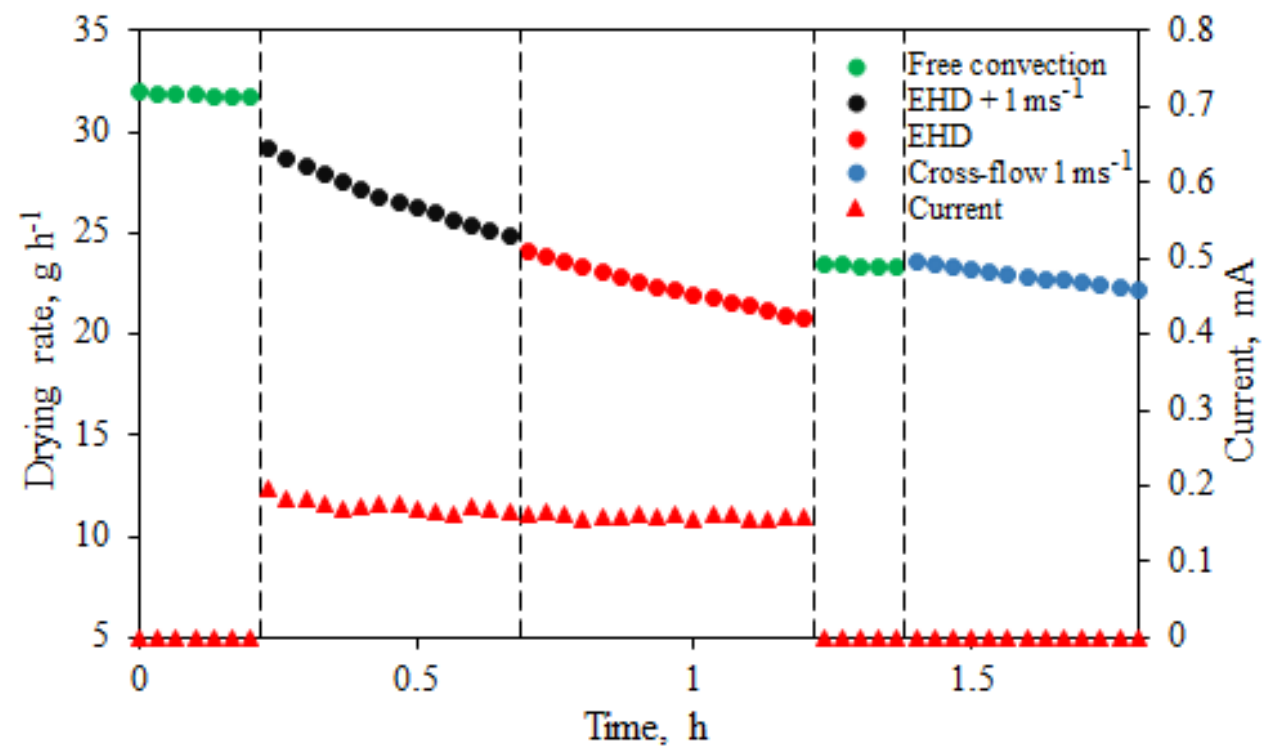

Fig. 6 An interactions between forced air cross-flow $\left(1 \mathrm{~m} \mathrm{~s}^{-1}\right)$ and electro-convection (18 kV) at $3.5 \mathrm{~cm}$ gap, $20 \pm 2 \%$ RH.

The case \# 3 was found to be the most efficient. The enhancement ratio was: EHD/Free convection $=6.66 / 1.4=4.757$, EHD/Cross-flow $=6.66 / 3.36=1.982$, EHD+Crossflow $/$ Free convection $=10.04 / 1.4=7.171$, EHD+Cross-flow $/$ Cross-flow $=10.04 / 3.36=$ 2.988. It follows that EHD effect was always positive, resulting in enhancement of drying rate in the range from 1.98 to 7.17 times.

\section{Conclusions}

1. EHD drying kinetics typically followed the exponential curve, hence it is diffusionlimited and could be described by Fick's equation.

2. Drying rate of white champignons is proportional to initial moisture content, increases with electric field strength and with decrease of relative humidity.

3. The effect of needle packing density on drying rate of white champignons was significant.

4. The effect of $1.0 \mathrm{~m} \mathrm{~s}^{-1}$ cross-flow air on EHD drying was found significant at 12 and 14 $\mathrm{kV}$, while at $16 \mathrm{kV}$ the drying rate was higher than EHD without air cross-flow.

5. Effect of electric field on effective moisture diffusion was much more pronounced in the case of sole EHD: from $3.38 \cdot 10^{-10} \mathrm{~m}^{2} \mathrm{~s}^{-1}(12 \mathrm{kV})$ to $7.22 \cdot 10^{-10} \mathrm{~m}^{2} \mathrm{~s}^{-1}(16 \mathrm{kV})$. 


\section{References}

[1] Kudra, T.; Martynenko, A. Energy aspects in electrohydrodynamic drying. Drying Technology 2015, 33, 1534-1540.

[2] Giri, S.K.; Prasad, S. Drying kinetics and rehydration characteristics of microwavevacuum and convective hot-air dried mushrooms. Journal of Food Engineering 2007, 78 (2), 512-521.

[3] Xue, G.R.; Limoto, M.; Uchino T. Drying of shiitake mushrooms using ionic wind generated by corona discharge. Journal of the Japan Society of Agricultural Machinery 1996, 58 (4), 53-60.

[4] Dutta, B.; Raghavan, G.S.V.; Dev, S.R.S.; Liplap, P.; Murugesan, R.; Anekella K.; Kaushal, T.A. Comparative study on the effects of microwave and high electric field pretreatments on drying kinetics and quality of mushrooms. Drying Technology 2012, 30 (8), 891-897.

[5] Dinani, S.T.; Havet, M.; Hamdami, N.; Shahedi, M. Drying of mushroom slices using hot air combined with an electrohydrodynamic (EHD) drying system. Drying Technology 2014a, 32 (5), 597-605.

[6] Dinani, S.T.; Hamdami, N.; Shahedi, M.; Havet, M. Mathematical modeling of hot air/electrohydrodynamic (EHD) drying kinetics of mushroom slices. Energy Conversion and Management 2014b, 86, 70-80.

[7] Dinani, S.T.; Havet, M. Drying kinetics and energy consumption of combined convective-electrohydrodynamic (EHD) drying of mushroom slices. In 19-th International Drying Symposium. Lyon, France. August 24-27, 2014c.

[8] Dinani, S.T.; Havet, M. The influence of voltage and air flow velocity of combined convective-electrohydrodynamic drying system on the kinetics and energy consumption of mushroom slices. Journal of Cleaner Production 2015a, 95, 203211.

[9] Dinani, S.T.; Hamdami, N.; Shahedi, M.; Havet, M.; Queveau, D. Influence of the electrohydrodynamic process on the properties of dried button mushroom slices: A differential scanning calorimetry (DSC) study. Food and Bioproducts Processing 2015b, 95, 83-95.

[10] Singh, A.; Vanga S.K.K.; Nair, G.R.; Gariepy Y.; Orsat, V., Raghavan, V. Electrohydrodynamic drying of sand. Drying Technology 2017, 35 (3), 312-322.

[11] Argyropoulos, D.; Rainer, A.; Mueller, J. Establishing moisture sorption isotherms of wild mushroom varieties using a dynamic vapor sorption method. XVII-th World Congress of the International Commission of Agricultural and Biosystems Engineering (CIGR). Québec City, Canada June 13-17, 2010.

[12] Labuza, T.P.; McNally, L.; Gallagher, D.; Hawkes, J.; Hurtado, F. Stability of intermediate moisture foods. 1. Lipid Oxidation. Journal of Food Science 1979, 37, 154-159. 